\section{Perfil epidemiológico do beribéri notificado de 2006 a 2008 no Estado do Maranhão, Brasil}

\author{
Epidemiological profile of reported beriberi cases \\ in Maranhão State, Brazil, 2006-2008
}

\section{Abstract}

This study aimed to describe the epidemiological profile of beriberi cases and related deaths reported from 2006 to 2008 in Maranhão State, Brazil. Data were obtained from beriberi notification forms at the State Health Department. The global Moran index was used to evaluate spatial auto-correlation. 1207 cases and 40 deaths were reported. The western and central regions of the State showed strong spatial auto-correlation of incidence rates. Cases and deaths were concentrated from May to August, in young men (20-40 years). Regular alcohol consumption and smoking were recorded among fatal cases. Low income and heavy labor were widespread among cases. Common symptoms were asthenia, numbness, and swollen legs, difficulty walking, and calf pain. The profile of cases and their symptoms (except swollen legs) are characteristic of dry beriberi. We recommend further studies on the resurgence of beriberi in Brazil.

Beriberi; Thiamine Deficiency; Health Profile

\author{
Estela Maura Padilha ${ }^{1}$ \\ Elizabeth Fujimori 2 \\ Ana Luiza Vilela Borges 2 \\ Ana Paula Sayuri Sato 2 \\ Murilo Novaes Gomes 3 \\ Maria dos Remédios Freitas Carvalho Branco 4 \\ Henrique Jorge dos Santos 5 \\ Nulvio Lermen Junior 1
}

\section{Introdução}

O beribéri é uma doença de natureza carencial, causada por deficiência de tiamina, que, apesar de facilmente tratável, pode levar a óbito. A tiamina é uma vitamina hidrossolúvel do complexo B (B1), da qual o organismo humano não consegue manter reservas duradouras, e, se não houver ingestão diária, a saturação tecidual tem duração de, no máximo, dois a três meses 1 .

Entre as causas associadas à deficiência de tiamina, destaca-se a alimentação monótona, com base no consumo de arroz polido, ingestão elevada de carboidratos simples e consumo de bebidas alcoólicas. Substâncias com efeito antitiamina e contaminação de alimentos por micotoxinas também têm sido associadas ao desenvolvimento do beribéri 1,2 .

Durante os últimos três séculos, surtos da doença têm sido descritos em diferentes regiões do mundo, principalmente relacionados a hábitos alimentares e situações de insegurança alimentar, restrição e/ou privação ${ }^{1}$. Nas últimas décadas, há relatos de ocorrência em Gâmbia 3,4; Cuba 5; Colômbia 6; Costa do Marfim 7 e Tailândia 8 , sempre associada à alimentação deficiente (restrita).

No Brasil, registros de epidemia de beribéri datam do final do século XVII. Há pelo menos 80 anos não se tinha referência de surtos de beribéri no país, tendo o último ocorrido em um asilo, entre o final do século XIX e início do século XX 9 . 
No entanto, casos recentes têm sido notificados a partir de 2006 nos estados do Maranhão, Tocantins e Roraima, com hipóteses diagnósticas iniciais de doença de etiologia desconhecida, intoxicação por agrotóxicos ou por bebida alcoólica e síndrome de Guillain-Barré 10.

Tendo em vista que pouco se sabe sobre o atual surto de beribéri no país, sua magnitude no Estado do Maranhão e o perfil dos acometidos, este estudo descreve características epidemiológicas dos casos de beribéri e óbitos notificados no Estado do Maranhão no período de 2006 a 2008.

\section{Método}

Este estudo descritivo e retrospectivo foi desenvolvido junto à Secretaria de Estado da Saúde do Maranhão (SESMA).

O Maranhão é um estado localizado na Região Nordeste do Brasil. Possui 217 municípios distribuídos em uma área de $331.935 \mathrm{~km}^{2}$ e densidade demográfica de 19,7 habitantes $/ \mathrm{km}^{2}$. Em 2009, a população estimada era de 6.569 .683 habitantes, distribuída em $63 \%$ na área urbana e $37 \%$ na área rural 11. A economia é baseada principalmente na indústria de transformação de alumínio, alimentícia, madeireira, no extrativismo (babaçu), agricultura (arroz, milho, soja, mandioca), pecuária e serviços. A síntese dos principais indicadores sociais mostra que 56\% de sua população é pobre, sendo a unidade federativa do Nordeste que apresenta a menor renda domiciliar per capita e a prevalência mais elevada de insegurança alimentar grave 12. Em 2005, o Índice de Desenvolvimento Humano (IDH) do Estado do Maranhão era de 0,636, ocupando o vigésimo sexto lugar no ranking nacional ${ }^{13}$. Na maior parte do território, o clima é tropical, com chuvas distribuídas nos primeiros meses do ano, porém o oeste maranhense é caracterizado por clima equatorial, com médias pluviométricas e térmicas elevadas, de janeiro a maio (Instituto Brasileiro de Geografia e Estatística. SIDRA - Sistema IBGE de Recuperação Automática. http://www.sidra.ibge.gov.br, acessado em 17/ Mai/2010). As informações dos casos de beribéri e óbitos notificados no período de 2006 a 2008 foram obtidas no banco de dados da vigilância epidemiológica da SESMA. Tendo em vista que o banco continha muitas variáveis em branco, as informações foram complementadas por meio de consulta às fichas de notificação/investigação de Beribéri. No total, $72 \%$ das fichas foram analisadas, sendo 95\% de 2006, 61\% de 2007 e 54\% de 2008. Apesar da verificação individual desses documentos, nem todos os campos tinham sido preenchidos, de modo que algumas informações importantes, como renda familiar, consumo de álcool e hábito de fumar, praticamente se tornaram irrecuperáveis ao se considerar o custo para a realização de visitas domiciliares para a obtenção desses dados.

É importante destacar que o modelo da ficha de notificação/investigação adotado em 2006 não contemplou informações referentes à escolaridade, ocupação, renda familiar e forma clínica. Logo, não foi possível avaliar essas variáveis para os casos e óbitos notificados em 2006 e para alguns casos de 2007 e 2008 que foram notificados com a utilização do modelo de 2006.

A distribuição espacial da incidência do beribéri entre os municípios foi analisada por meio do Índice Global de Moran e Indicador Local de Associação Espacial 14. O Índice de Moran fornece uma medida geral da associação espacial existente no conjunto dos dados. Seu valor varia de - 1 a 1 . Valores próximos de zero indicam a inexistência de autocorrelação espacial significativa dos valores de incidência de beribéri entre municípios e seus vizinhos. Valores positivos para o índice indicam autocorrelação espacial positiva, ou seja, o valor da incidência de um município tende a ser semelhante aos valores dos seus vizinhos. Valores negativos para o índice, por sua vez, indicam autocorrelação negativa. A autocorrelação espacial é definida como a propriedade de variáveis aleatórias possuírem valores que são mais similares ou menos similares do que o esperado para pares de observações aleatoriamente associados, em virtude de uma existente proximidade geográfica. O Indicador Local de Associação Espacial produz um valor específico para cada município, permitindo, assim, a identificação de agrupamentos de municípios com valores de incidência semelhantes (clusters), aqui demonstrados pelo mapa de espalhamento de Moran (Box Map). Esse mapa identifica municípios cujos vizinhos apresentam valores de incidência de beribéri semelhantes; desse modo, o mapa de espalhamento é construído por categorias, sendo possível duas classes de associação espacial positiva (Alto-Alto; Baixo-Baixo) e duas de associação espacial negativa (Alto-Baixo; Baixo-Alto).

Os casos e óbitos foram ainda analisados segundo quadrimestre de ocorrência, área de residência do acometido (rural ou urbana), forma clínica (seco, úmido, shoshin, síndrome de Wernicke-Korsakoff e associadas), características socioeconômico-demográficas (sexo, idade, escolaridade, ocupação e renda familiar mensal) e variáveis relacionadas à doença (tempo decorrido entre os primeiros sintomas e a notificação, hospitalização, consumo de bebida alcoólica e hábito de fumar). 
Para a ocupação, foi utilizada a categorização com base no gasto energético, proposta por Gomes et al. 15: leve (exercida sentada, com movimentos leves de braços e tronco ou exercidas em pé, com trabalho leve em máquina ou bancada e movimento leve de braços e pernas); moderada (exercida em pé, com trabalho leve em máquinas ou bancada e movimentação vigorosa de braços e pernas); pesada (trabalho intermitente de levantar ou arrastar). Desta forma, foram categorizadas como atividades leves - estudante, motorista, funcionário público, professor, comerciante, locutor de rádio, aposentado, guardanoturno e desempregado; atividades moderadas - dona de casa/do lar, doméstica, babá, serviços gerais, diarista, lavadeira, auxiliar de saúde, moveleiro, auxiliar de cozinha, ambulante, sorveteiro, pescador, vigilante, caseiro, agente de saúde, açougueiro, lavador de carro, gráfico, lanterneiro, mecânico, estoquista, barbeiro, auxiliar administrativo, marceneiro e ceramista; atividades pesadas - lavrador, serralheiro, pedreiro, vaqueiro, agricultor, servente, ajudante de pedreiro, cavador de açude, tratorista, estivador e operador de motosserra.

Os dados foram descritos por meio de proporções e médias no programa SPSS, versão 15.0 (SPSS Inc., Chicago, Estados Unidos); para análise espacial, foi utilizado o Terraview 3.2.0 RC1 (Instituto Nacional de Pesquisas Espaciais; http://www.dpi.inpe.br/terraview/índex.php). Por se tratar de investigação com base em dados secundários, na qual os sujeitos da pesquisa não foram abordados, o Comitê de Ética em Pesquisa ao qual este estudo foi submetido considerou não haver implicações éticas. Entretanto, seguindo as normas exigidas pela Resolução no. 196/96, obteve-se autorização da SESMA e do Ministério da Saúde para utilização do banco de dados e realização das análises efetuadas. Além disso, este estudo foi realizado sem recursos financeiros institucionais e/ou privados.

\section{Resultados}

No período de 2006 a 2008, 1.207 casos e 40 óbitos por beribéri foram notificados no Maranhão, tendo todos os óbitos ocorrido no ano de 2006.

Mais de um quarto dos municípios do estado registraram ocorrência de casos $(n=57 ; 26,2 \%)$, 22 dos quais $(38,6 \%)$ apresentaram casos nos três anos analisados, e 17 (29,8\%) registraram apenas um caso em um dos anos estudados. Os óbitos ocorreram em 21 municípios.

O perfil dos casos e óbitos apresentados na Tabela 1 mostra que quase a totalidade dos óbitos ocorreu entre indivíduos do sexo masculino, a grande maioria na faixa etária compreendida entre 20 e 30 anos (72,5\%), que residiam em área urbana (80\%). Para dois terços dos que foram a óbito, o tempo decorrido entre os primeiros sintomas e a notificação foi inferior a três meses; a hospitalização ocorreu para $42,5 \%$ e a proporção dos que referiram hábito de consumir álcool e fumar foi elevada, $75 \%$ e $66,7 \%$, respectivamente. Em relação à distribuição sazonal, verificou-se que todos os óbitos ocorreram entre os meses de maio e agosto.

Indivíduos do sexo masculino e a faixa etária de 20 a 40 anos foram os mais acometidos. $\mathrm{O}$ tempo decorrido entre os primeiros sintomas e a notificação foi inferior a três meses para cerca de dois terço dos casos. Percentual elevado das fichas de notificação não continha informações referentes a hospitalização, consumo de álcool e hábito de fumar, de forma que esses dados devem ser analisados com cautela. Entre as fichas com tais informações, verificou-se que a taxa de hospitalização diminuiu gradativamente no período. O consumo de álcool foi referido por quase metade dos acometidos e o hábito de fumar, por cerca de um terço. Verificou-se que mais da metade tinha quatro anos ou menos de estudo, a ocupação de dois terços era relacionada a atividade pesada e quase três quartos tinham renda familiar inferior a um salário mínimo. Em relação ao local de residência, verificou-se predomínio da zona urbana (57,6\%). O beribéri seco foi a forma clínica predominante, porém não foi possível obter essa informação para os óbitos, uma vez que o modelo da ficha de notificação elaborada para o ano de 2006 não incluía essa informação. Análise da distribuição sazonal mostrou que a ocorrência dos casos de beribéri concentrou-se entre os meses de maio e agosto, da mesma forma que os óbitos (Tabela 1).

Os sintomas mais referidos, informados nas fichas de notificação, foram: diminuição da força nas pernas, dormência e edema de membros inferiores, dificuldade para caminhar e dor na panturrilha. Dispneia e cansaço também foram relatados, mas os dados não estão apresentados em tabela.

Na Tabela 2, são apresentadas as médias dos coeficientes de incidência dos casos de beribéri notificados entre 2006 e 2008 por município. Nesse período, o coeficiente de incidência na população do Maranhão foi de 4,32/10 mil habitantes. Os municípios que apresentaram os maiores coeficientes de incidência nos três anos analisados foram São João do Caru (12,90/10 mil habitantes), João Lisboa (11,23/10 mil habitantes) e São João do Paraíso (10,09/10 mil habitantes).

O valor do Índice Global de Moran (I Moran = 0,43 ) confirmou autocorrelação espacial da inci- 
Distribuição dos óbitos e casos notificados de beribéri, segundo características socioeconômico-demográficas e relacionadas à doença. Maranhão, Brasil, 2006 a 2008.

\begin{tabular}{|c|c|c|c|c|}
\hline \multirow[t]{2}{*}{ Características } & \multicolumn{2}{|c|}{ Óbitos } & \multicolumn{2}{|c|}{ Casos } \\
\hline & $\mathrm{n}$ & $\%$ & $n$ & $\%$ \\
\hline \multicolumn{5}{|l|}{ Sexo } \\
\hline Masculino & 39 & 97,5 & 979 & 81,9 \\
\hline Feminino & 1 & 2,5 & 216 & 18,1 \\
\hline \multicolumn{5}{|l|}{ Faixa etária (anos) } \\
\hline$<20$ & 8 & 20,2 & 216 & 18,2 \\
\hline $20 \mid-30$ & 29 & 72,5 & 423 & 36,6 \\
\hline $30 \mid-40$ & 3 & 7,2 & 260 & 21,9 \\
\hline$\geq 40$ & 0 & 0,0 & 288 & 24,3 \\
\hline \multicolumn{5}{|l|}{ Tempo decorrido (meses) * } \\
\hline$<3$ & 26 & 65,0 & 692 & 69,8 \\
\hline 3 e mais & 14 & 35,0 & 342 & 34,5 \\
\hline \multicolumn{5}{|l|}{ Hospitalização } \\
\hline Sim & 17 & 42,5 & 285 & 35,9 \\
\hline Não & 23 & 57,5 & 509 & 64,1 \\
\hline \multicolumn{5}{|l|}{ Consumo de álcool } \\
\hline Sim & 12 & 75,0 & 364 & 45,9 \\
\hline Não & 4 & 25,0 & 429 & 54,1 \\
\hline \multicolumn{5}{|l|}{ Hábito de fumar } \\
\hline Sim & 10 & 66,7 & 279 & 35,1 \\
\hline Não & 5 & 33,3 & 516 & 64,9 \\
\hline \multicolumn{5}{|l|}{ Escolaridade (anos) } \\
\hline 이-5 & $\star \star$ & $\star \star$ & 255 & 57,2 \\
\hline $5 \mid-9$ & $\star \star$ & $\star \star$ & 131 & 29,4 \\
\hline $9 \mid-12$ & $\star \star$ & $\star \star *$ & 42 & 9,4 \\
\hline$\geq 12$ & $\star \star$ & $\star \star$ & 18 & 4,0 \\
\hline \multicolumn{5}{|l|}{ Ocupação (atividade) } \\
\hline Leve & $\star \star$ & $\star \star$ & 91 & 16,4 \\
\hline Moderada & $\star \star$ & $\star \star *$ & 96 & 17,3 \\
\hline Pesada & $\star \star$ & $\star \star$ & 367 & 66,3 \\
\hline \multicolumn{5}{|c|}{ Renda familiar (salários mínimos) } \\
\hline$<1$ & $\star \star$ & ** & 331 & 72,9 \\
\hline $1-2$ & $\star \star$ & $\star \star$ & 56 & 12,4 \\
\hline$>2$ & $\star \star$ & $\star \star$ & 67 & 14,7 \\
\hline \multicolumn{5}{|l|}{ Área de residência } \\
\hline Urbana & 12 & 80,0 & 468 & 57,6 \\
\hline Rural & 3 & 20,0 & 345 & 42,4 \\
\hline \multicolumn{5}{|l|}{ Forma clínica } \\
\hline Seco & $\star \star$ & $\star \star$ & 654 & 87,4 \\
\hline Úmido & $\star \star$ & $\star \star$ & 52 & 6,9 \\
\hline Shoshin & $\star \star$ & $\star \star$ & 6 & 0,8 \\
\hline Associadas & & & 36 & 4,5 \\
\hline \multicolumn{5}{|l|}{ Mês de notificação } \\
\hline Janeiro a abril & 0 & 0,0 & 294 & 25,2 \\
\hline Maio a agosto & 40 & 100,0 & 705 & 60,5 \\
\hline Setembro a dezembro & 0 & 0,0 & 167 & 14,3 \\
\hline
\end{tabular}

* Tempo decorrido entre os primeiros sintomas e a notificação;

** Dados não disponíveis. 
Coeficiente de incidência por 10.000 habitantes, segundo municípios e faixa de incidência. Maranhão, Brasil, 2006 a 2008.

\begin{tabular}{|c|c|}
\hline Municípios & Coeficiente de incidência (por 10.000 habitantes) \\
\hline \multicolumn{2}{|l|}{ Faixa de incidência: $>8,40$} \\
\hline São João do Caru & 12,90 \\
\hline João Lisboa & 11,23 \\
\hline São João do Paraíso & 10,09 \\
\hline Amarante do Maranhão & 9,14 \\
\hline \multicolumn{2}{|l|}{ Faixa de incidência: $8,40-\mid 4,37$} \\
\hline Montes Altos & 8,39 \\
\hline Governador Newton Belo & 8,09 \\
\hline Senador La Roque & 7,15 \\
\hline Davinópolis & 6,45 \\
\hline Santa Luzia & 5,73 \\
\hline Buritirana & 5,64 \\
\hline \multicolumn{2}{|l|}{ Faixa de incidência: 4,37-|2,81 } \\
\hline Governador Edison Lobão & 4,37 \\
\hline Bom Jesus das Selvas & 3,87 \\
\hline Lajeado Novo & 3,87 \\
\hline Vila Nova dos Martírios & 3,81 \\
\hline Alto Alegre do Pindaré & 3,43 \\
\hline Cidelândia & 3,40 \\
\hline Fernando Falcão & 3,38 \\
\hline \multicolumn{2}{|l|}{ Faixa de incidência: $2,81-\mid 1,92$} \\
\hline Imperatriz & 2,81 \\
\hline Centro Novo do Guilherme & 2,68 \\
\hline Porto Franco & 2,58 \\
\hline Bom Jardim & 2,47 \\
\hline \multicolumn{2}{|l|}{ Faixa de incidência: 1,92-|0,79 } \\
\hline Grajaú & 1,92 \\
\hline São Pedro da Água Branca & 1,79 \\
\hline Açailândia & 1,24 \\
\hline Estreito & 1,21 \\
\hline Itinga do Maranhão & 1,19 \\
\hline \multicolumn{2}{|l|}{ Faixa de incidência: $0,79-\mid 0,54$} \\
\hline Sítio Novo & 0,79 \\
\hline Olho d'Água das Cunhãs & 0,78 \\
\hline Jatobá & 0,77 \\
\hline Monção & 0,75 \\
\hline Centro do Maranhão & 0,67 \\
\hline Barra do Corda & 0,65 \\
\hline \multicolumn{2}{|l|}{ Faixa de incidência: $0,54-\mid 0,32$} \\
\hline Graça Aranha & 0,54 \\
\hline Governador Luiz Rocha & 0,52 \\
\hline Amapá do Maranhão & 0,50 \\
\hline São Francisco do Brejão & 0,40 \\
\hline São João do Soter & 0,37 \\
\hline
\end{tabular}

(continua)

dência do beribéri (Figura 1). Em relação ao mapa de espalhamento, observou-se, principalmente, uma distribuição espacial com alta incidência na parte oeste e central do estado (Alto-Alto), uma zona de transição de municípios com baixa incidência margeados por municípios com altos valores de incidência (Baixo-Alto) e, por fim, uma grande região norte, sul e leste formando clusters 
Tabela 2 (continuação)

\begin{tabular}{lc} 
Municípios & Coeficiente de incidência (por 10.000 habitantes) \\
\hline Faixa de incidência: 0,32-|0,16 & \\
Santa Luzia do Paruá & 0,32 \\
Bela Vista do Maranhão & 0,32 \\
Jenipapo dos Vieiras & 0,23 \\
Pindaré-Mirim & 0,23 \\
Buriticupu & 0,22 \\
Nova Olinda & 0,19 \\
Formosa da Serra Negra & 0,19 \\
Faixa de incidência: 0,16-|0,01 & \\
Riachão & 0,16 \\
Mirador & 0,15 \\
Campestre do Maranhão & 0,14 \\
Carolina & 0,14 \\
Buriti & 0,13 \\
Santa Inês & 0,13 \\
Zé Doca & 0,11 \\
Vitorino Freire & 0,11 \\
Governador Nunes Freire & 0,09 \\
Arame & 0,05 \\
Balsas & 0,05 \\
Paço do Lumiar & 0,03 \\
São Luís & 0,02 \\
Maranhão & 4,32 \\
\hline
\end{tabular}

de municípios com baixos valores de incidência (Baixo-Baixo).

No período estudado, a taxa de mortalidade do beribéri na população do Maranhão foi de 0,45 por 10 mil habitantes. Os municípios com as maiores taxas de mortalidade foram Davinópolis, Vila Nova dos Martírios e São Pedro da Água Branca, conforme Tabela 3.

\section{Discussão}

Em nível mundial, o beribéri não é mais uma doença largamente difundida na população. Apenas focos isolados têm sido observados nos últimos 20 anos entre refugiados e comunidades restritas, como a que acometeu detentos de uma penitenciária na Costa do Marfim 7 , tripulações de navios na Tailândia ${ }^{8}$, refugiados butaneses no Nepal ${ }^{1}$ e fuzileiros navais na Colômbia 6 .

O padrão geográfico de ocorrência do beribéri no Maranhão, que afetou cerca de um quarto dos municípios do estado, localizados nas regiões oeste e central, é atípico. Essa distribuição espacial, no entanto, guarda certa semelhança com a epidemia de neuropatia - também possivelmente associada à deficiência de vitaminas do complexo B - que afetou mais de 50 mil cuba- nos no período de 1991-1993. Os casos em Cuba iniciaram-se em uma província rural no extremo oeste da ilha e se estenderam por praticamente todo o país, com um padrão de distribuição geográfica do oeste para o leste; no entanto, a distribuição mostrou-se bastante heterogênea, com municípios apresentando poucos casos, mesmo em províncias com elevado coeficiente de incidência 5,16. No presente estudo, constatou-se padrão semelhante ao cubano, pois, do total de municípios afetados, $30 \%$ apresentaram um único caso no ano.

A forte dependência espacial - clusters de municípios com presença da enfermidade a oeste e centro do estado e ausência de casos no norte, leste e sul - indica que as regiões oeste e central do estado possuem certas características geográficas que as diferenciam do resto do estado e que propiciaram o surgimento dos casos de beribéri.

O Estado do Maranhão apresenta certa homogeneidade espacial em relação aos principais índices sociais, como o IDH, a renda per capita, a taxa de alfabetização, a esperança de vida ao nascer, entre outros (Instituto Brasileiro de Geografia e Estatística. Estado do Maranhão. http:// www.ibge.gov.br/estadoat/perfil.php?sigla=ma, acessado em 17/Mai/2010). A homogeneidade espacial também ocorre em relação à área pro- 

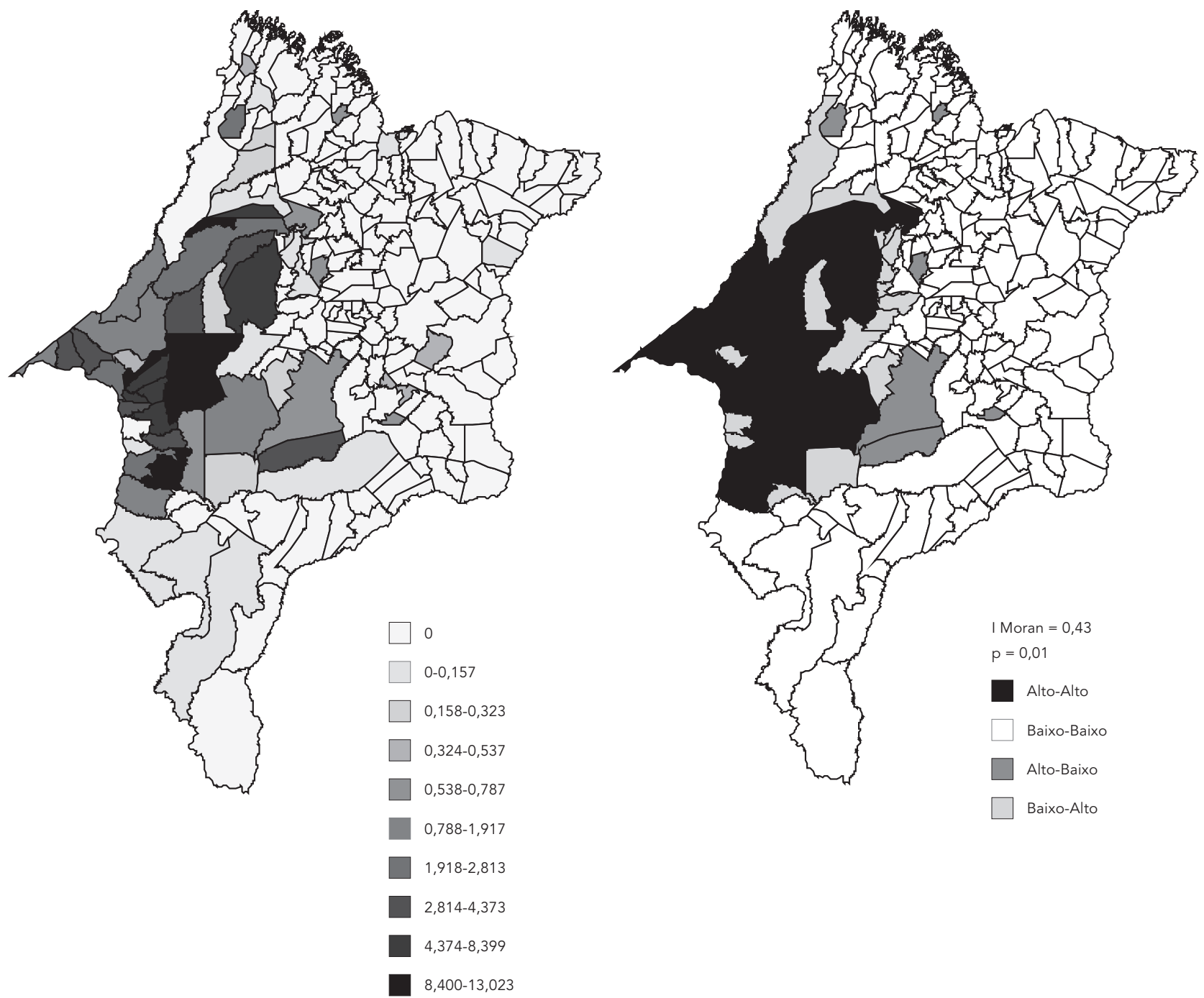

dutora de arroz por município (Instituto Brasileiro de Geografia e Estatística. SIDRA - Sistema IBGE de Recuperação Automática. http://www. sidra.ibge.gov.br, acessado em 17/Mai/2010), principal cultura agrícola que pode estar associada à enfermidade. Portanto, há necessidade de se investigarem, com maior profundidade, quais são os elementos associados ao beribéri que estão presentes nas regiões oeste e central do estado, mas ausentes nas demais. A presença de municípios com baixos valores de incidência no cluster dos municípios com altos valores (BaixoAlto) pode se dever à não realização de busca ativa de casos, reforçada pelo fato de estas cidades não terem sido alvo das ações de enfrentamento implementadas nos demais municípios pela força tarefa interministerial.

De qualquer forma, a suspeita de contaminação do arroz por micotoxinas de fungos foi levantada como possível etiologia do ressurgimento do beribéri no Maranhão 10, uma vez que as áreas com casos notificados são produtoras de arroz; 
Taxa de mortalidade por beribéri (por 10.000 habitantes), segundo município. Maranhão, Brasil, 2006.

\begin{tabular}{|c|c|}
\hline Município & Taxa \\
\hline Davinópolis & 3,37 \\
\hline Vila Nova dos Martírios & 2,54 \\
\hline São Pedro da Água Branca & 1,79 \\
\hline Porto Franco & 1,66 \\
\hline Lajeado Novo & 1,49 \\
\hline Montes Altos & 0,90 \\
\hline São João do Paraíso & 0,84 \\
\hline Sítio Novo & 0,59 \\
\hline Senador La Roque & 0,52 \\
\hline João Lisboa & 0,49 \\
\hline Santa Luzia & 0,44 \\
\hline Bom Jesus das Selvas & 0,43 \\
\hline Grajaú & 0,34 \\
\hline Arame & 0,33 \\
\hline Itinga do Maranhão & 0,32 \\
\hline Imperatriz & 0,30 \\
\hline Amarante do Maranhão & 0,26 \\
\hline Bom Jardim & 0,24 \\
\hline Açailândia & 0,19 \\
\hline Buritirana & 0,13 \\
\hline Buriticupu & 0,13 \\
\hline Total & 0,45 \\
\hline
\end{tabular}

assim, a doença poderia acometer tanto os trabalhadores rurais, quanto os consumidores 17.

No Japão, surtos de beribéri foram associados à contaminação do arroz pela micotoxina citreoviridina, que interfere na absorção da tiamina 18 . Apesar das providências adotadas, como a interdição cautelar do arroz suspeito e a substituição do arroz interditado, ainda não foi possível associar a presença de fator tóxico ao quadro clínico 19 , o que permite concluir que a dieta restrita ao arroz polido e a baixa condição socioeconômica da maioria dos acometidos podem estar, de fato, associadas ao surto de beribéri aqui discutido.

O padrão sazonal verificado, com ocorrência de maior percentual de casos e óbitos no período de maio a agosto, também pode estar relacionado às atividades agrícolas na região, em que o sistema de cultivo predominante é de arroz, milho, mandioca e feijão. Tal período coincide com o final do plantio de arroz e início do plantio de outras culturas, como, por exemplo, a do feijão 20 . A atividade agropecuária é classificada como atividade pesada, que eleva marcadamente o requerimento energético e, em consequência, a exigência de tiamina. Assim, indivíduos que já subsistem com dietas pobres em tiamina, podem desenvolver sintomas de deficiência dessa vitamina quando se submetem à atividade física intensa ${ }^{1}$.

Em Gâmbia, os surtos de beribéri também apresentaram distribuição temporal, porém ocorreram logo após 4 ou durante a segunda metade da estação chuvosa 3 , o que coincidia com baixo suprimento alimentar e atividade agrícola intensa. Apesar da associação com a intensa atividade agrícola, a maioria dos acometidos de Gâmbia residia em área urbana 3 , estando em conformidade com o verificado no presente estudo, muito provavelmente porque, embora residissem em área urbana, a grande maioria trabalhava na agricultura.

O perfil dos acometidos, isto é, mais homens e jovens, na faixa de 20 a 40 anos de idade, é similar ao verificado em outros estudos 4,6,7,8. A justificativa é que o sexo masculino predomina nos confinamentos como prisões ou tripulações marinhas; some-se a isso o fato de que são os homens que mais frequentemente se envolvem em ocupações que requerem maior gasto energético e esforço físico.

Em relação aos sinais e sintomas, de forma geral, há dificuldade para se estabelecer a sintomatologia clínica específica da deficiência de tiamina. Isso se deve às amplas variações de- 
correntes da presença de fatores complicadores, como presença de múltiplas deficiências de vitaminas e minerais, bem como aos efeitos de outros aspectos, como trabalho físico pesado, gravidez e infecções 1 . Todavia, à exceção do edema de membros inferiores, os principais sinais e sintomas encontrados, diminuição da força nas pernas, dormência dos membros inferiores, dor na panturrilha e dificuldade para caminhar, são próprios do beribéri seco, o qual se caracteriza por polineurite, com parestesia das extremidades, especialmente das pernas, e progressiva fraqueza 1 . Tais sinais e sintomas também são os mais referidos na literatura, porém dispneia, cansaço, parestesia e edema de membros inferiores, presentes em muitos casos, são compatíveis com o beribéri úmido, configurando a forma combinada de beribéri seco e úmido ${ }^{2}$. O beribéri seco, no entanto, foi a forma clínica predominante nos casos notificados em 2007 e 2008. Além disso, a ficha de notificação de 2006 não incluía esta informação, mas dados da Secretaria de Vigilância à Saúde do Ministério da Saúde indicam que a forma clínica shoshin foi responsável pela quase totalidade dos óbitos 21 .

Como, no início, a sintomatologia é inespecífica, há dificuldade para o diagnóstico precoce do beribéri, que geralmente ocorre somente quando os sintomas evoluem para quadros mais graves ${ }^{1}$. Assim, é possível que os maranhenses acometidos praticamente não dispusessem da pequena reserva que o organismo mantém de vitaminas hidrossolúveis, uma vez que o tempo decorrido entre o aparecimento dos primeiros sintomas e a notificação foi inferior a três meses para a grande maioria dos casos e óbitos. Esse curto intervalo de tempo também sugere êxito na capacitação dos profissionais de saúde no que se refere ao diagnóstico do problema. Da mesma forma, a diminuição progressiva da hospitalização dos casos notificados pode ter sido decorrente das ações de enfrentamento implementadas pela força tarefa interministerial, que congregou órgãos estaduais e municipais ao longo de 2007 e 2008 para o controle da doença 22.

O consumo de álcool e o hábito de fumar foram relativamente altos entre os indivíduos classificados como casos, mas muito mais elevado entre os que foram a óbito. Em Cuba, constatou-se associação significativa entre a neuropatia epidêmica de 1991-1993 e o hábito de fumar, indicando que o fator tóxico do fumo, associado a deficiências nutricionais, poderia facilitar a expressão clínica da enfermidade 5,16. Ademais, inúmeros estudos têm evidenciado associação entre alcoolismo e fumo à deficiência de tiamina $1,2,16,23,24$.

Como a maioria das doenças nutricionais, grande parte dos surtos de beribéri associa-se a condições de pobreza e fome, consumo de alimentação monótona baseada em arroz polido, elevado teor de carboidratos simples e também a alguns grupos de risco específicos, como aqueles engajados em atividade física pesada 2,4,7,23,25.

Dessa forma, apesar do elevado percentual de incompletude das informações, não causa surpresa que a baixa renda familiar estivesse presente em quase três quartos dos casos notificados. Essa condição, aliada à baixa escolaridade dos indivíduos acometidos, que dificulta a inserção em trabalhos mais qualificados, ajuda a entender a alimentação deficiente e monótona como provável etiologia do beribéri. Dados da Pesquisa de Orçamentos Familiares de 2002/2003 indicaram que a aquisição domiciliar per capita de arroz no Maranhão supera as médias nacionais e do Nordeste 26 , reforçando a hipótese de dieta fortemente baseada no consumo desse alimento. Diante disso, as ações de enfrentamento do beribéri exigem intervenção intersetorial para o seu equacionamento, não podendo ficar restritas às ações de saúde.

Os resultados do presente estudo indicam que o perfil dos acometidos e os sinais e sintomas apresentados, à exceção do edema de membros inferiores, são característicos de beribéri seco. Os casos e óbitos concentraram-se no oeste do estado e acometeu a população socialmente mais excluída, inserida em atividade laboral pesada e com baixa renda e escolaridade. Logo, reiterase a preocupação de estudiosos do problema na região, no que se refere à necessidade de se buscarem mais informações da epidemia na suas dimensões social e econômica, as quais comprometem o acesso aos alimentos e à qualidade de vida da população 10 .

Considera-se pertinente que estudos sobre a determinação do processo de adoecer e morrer em decorrência de beribéri e seu ressurgimento no país sejam aprofundados, tendo em vista que casos continuam a ser notificados no Maranhão e em outros estados das regiões Norte e Nordeste. 


\section{Resumo}

O objetivo do estudo foi descrever o perfil epidemiológico dos casos e óbitos de beribéri notificados de 20062008 no Estado do Maranhão, Brasil. Informações foram obtidas de fichas de notificação da Secretaria de Estado da Saúde do Maranhão e utilizou-se o Índice Global de Moran para avaliar autocorrelação espacial. Foram notificados 1.207 casos e 40 óbitos. Regiões oeste e central mostraram forte presença de autocorrelação espacial da incidência. Ocorrência de casos e óbitos concentrou-se de maio a agosto, em homens jovens (20-40 anos). Hábito de consumir álcool e fumar esteve presente entre os óbitos; baixa renda e ocupação com atividade pesada, entre os casos. Os sintomas mais comuns foram diminuição da força, dormência e edema das pernas, dificuldade para caminhar e dor na panturrilha. O perfil dos acometidos e os sintomas, exceto edema de membros inferiores, são característicos de beribéri seco. É pertinente que estudos sobre seu ressurgimento no país sejam aprofundados.

Beribéri; Deficiência de Tiamina; Perfil de Saúde

\section{Colaboradores}

E. M. Padilha participou da concepção da pesquisa e do artigo, coordenou a coleta e o processamento dos dados e participou de todas as fases até a redação final. E. Fujimori contribuiu com a concepção da pesquisa e do artigo, orientou todas as etapas de realização e avaliação do trabalho e foi responsável pela redação final e revisão do artigo. A. L. V. Borges participou da análise de conteúdo, revisão e redação final do manuscrito. A. P. S. Sato contribuiu com a análise e interpretação dos dados e redação final do manuscrito. M. N. Gomes realizou a análise espacial e contribuiu com a interpretação dos dados e revisão final. M. R. F. C. Branco elaborou o projeto de pesquisa e contribuiu com a redação do manuscrito. H. J. Santos e N. Lermen Junior participaram da revisão final do artigo.

\section{Agradecimentos}

Agradecemos à Superintendência de Epidemiologia, Núcleo de Vigilância de Doenças e Agravos Não Transmissíveis (DANTS) da Secretaria de Estado da Saúde do Maranhão e ao Departamento de Atenção Básica do Ministério da Saúde, por disponibilizarem o banco de dados e relatórios, o que permitiu a realização deste trabalho.

\section{Referências}

1. World Heath Organization. Thiamine deficiency and its prevention and control in major emergencies. Geneva: World Heath Organization; 2006.

2. Lonsdale D. A review of the biochemistry, metabolism and clinical benefits of thiamin(e) and its derivatives. Evid Based Complement Alternat Med 2006; 3:49-59.

3. Rolfe M, Walker R, Samba K, Cham K. Urban beriberi in The Gambia, West Africa. Trans R Soc Trop Med Hyg 1993; 87:114-5.

4. Tang CM, Wells JC, Rolf M, Cham K. Outbreak of beriberi in the Gambia. Lancet 1989; 2:206-7.

5. Román GC. An epidemic in Cuba of optic neuropathy, sensorineural deafness, peripheral sensory neuropathy and dorsolateral myeloneuropathy. J Neurol Sci 1994; 127:11-28.

6. Martínez M, Román G, De La Hoz F, Podlesky E, Toro G, Osorio E, et al. Estudio clínico y epidemiológico de un brote de beriberi húmedo en Cartagena de Indias, Colombia, 1992-1993. Biomedica 1996; 16:41-51.
7. Ahoua L, Etiene W, Fermon F, Godain G, Brown V, Kadjo K, et al. Outbreak of beriberi in a prison in Côte d'Ivoire. Food Nutr Bull 2007; 28:283-90.

8. Doung-ngern P, Kesornsukhon S, Kanlayanaphotporn J, Wanadurongwan S, Songchitsomboon S. Beriberi outbreak among commercial fishermen, Thailand 2005. Southeast Asian J Trop Med 2007; 38:130-5.

9. Jacobina RR, Carvalho FM. Nina Rodrigues, epidemiologista: estudo histórico de surtos de beribéri em um asilo para doentes mentais na Bahia, 1897-1904. Hist Ciênc Saúde-Manguinhos 2001; 8:113-32.

10. Lira PIC, Andrade SLLS. Epidemia de beribéri no Maranhão, Brasil. Cad Saúde Pública 2008; 24:1202-3.

11. Instituto Brasileiro de Geografia e Estatística. Censo demográfico 2010 - primeiros resultados. Rio de Janeiro: Instituto Brasileiro de Geografia e Estatística; 2010. 
12. Instituto Brasileiro de Geografia e Estatística. Pesquisa Nacional por Amostra de Domicílio. Segurança alimentar - 2004. Rio de Janeiro: Instituto Brasileiro de Geografia e Estatística; 2006.

13. Instituto Brasileiro de Geografia e Estatística. Síntese de indicadores sociais. Estudos e pesquisas informação demográfica e socioeconômica. Rio de Janeiro: Instituto Brasileiro de Geografia e Estatística; 2006.

14. Anselin L. The Moran scatterplot as an ESDA tool to assess local instability in spatial association. In: Fischer M, Scholten HJ, Unwin D, editors. Spatial analytical perspectives on GIS in environmental and socio-economic sciences. London: Taylor \& Francis; 1996. p. 111-26.

15. Gomes VB, Siqueira KS, Sichieri R. Atividade física em uma amostra probabilística da população do Município do Rio de Janeiro. Cad Saúde Pública 2001; 17:969-76.

16. Rodríguez AP, García AI, Fernández I, Lago PM, Rodríguez AIG, Concepción AR. Posibles factores asociados con la neuropatía epidémica en Cuba. Rev Cuba Med Trop 1998; 50:54-60.

17. Instituto Brasileiro de Geografia e Estatística. Comunicação social: produção agrícola municipal - cereais, leguminosas e oleaginosas - 2002-2006. Rio de Janeiro: Instituto Brasileiro de Geografia e Estatística; 2007.

18. Ueno Y. Toxicology of microbial toxins. Pure Appl Chem 1986; 58:339-50.
19. Ministério da Agricultura, Pecuária e Abastecimento. Nota à imprensa: substituição de arroz no Maranhão http://www.planalto.gov.br/Consea/ exec/noticias.cfm?cod=19110 (acessado em 18/ Nov/2009).

20. Empresa Brasileira de Pesquisa Agropecuária. Embrapa adapta arroz de terras altas ao Sistema Plantio Direto. http://www.embrapa.gov.br/ imprensa/noticias/2005/marco/noticia.2005-0321.6240356157/ (acessado em 10/Jan/2010).

21. Secretaria de Vigilância em Saúde, Ministério da Saúde. Investigação de surto de beribéri na região Sudoeste do Estado do Maranhão, junho de 2006. Relatório final. Brasília: Ministério da Saúde; 2007.

22. Ministério da Saúde. Relatório final do Grupo Interministerial frente ao surto de beribéri na região sudoeste do Estado do Maranhão. Brasília: Ministério da Saúde; 2007.

23. Pearce JMS. Wernicke-Korsakoff encephalopathy. Eur Neurol 2008; 59:101-4.

24. Adamolekun B, Ndububa DA. Epidemiology and clinical presentation of a seasonal ataxia in western Nigeria. J Neurol Sci 1994; 124:95-8.

25. Krishna S, Taylor AM, Supanaranond W, Pukrittayakamee S, Kuile F, Tawfiq KM, et al. Thiamine deficiency and malaria in adults from Southeast Asia. Lancet 1999; 353:546-9.

26. Instituto Brasileiro de Geografia e Estatística. Pesquisa de Orçamentos Familiares 2002-2003: primeiros resultados. Brasil e grandes regiões. Rio de Janeiro: Instituto Brasileiro de Geografia e Estatística; 2004.

Recebido em 30/Jun/2010

Versão final reapresentada em 08/Dez/2010

Aprovado em 24/Jan/2011 